\title{
Mujeres y redes internacionales. La Liga Paraguaya Pro Derechos de la Mujer (1951-1962) como parte de las disputas de la Guerra Fría ${ }^{1}$
}

\author{
Women and international networks. The Paraguayan League for the Rights of Women (1951-1962) as part of the \\ Cold War disputes \\ Lorena Soler \\ Universidad de Buenos Aires - CONICET, Argentina \\ lorenamarinasoler@gmail.com
}

\section{Resumen:}

El artículo se inserta en la interacción problemática de la modernización política y las relaciones de género en el contexto de la Guerra Fría, para explicar las condiciones sociohistóricas que habilitaron el reconocimiento de los derechos políticos de las mujeres en Paraguay.Específicamente, se propone estudiar las formas asumidas parala conquista de la ciudadanía porun grupo de mujeresasuncenasorganizadas en la Liga Paraguaya Pro Derechos de la Mujer(1951-1962). Asume que dicha organización, a diferencia de otras experiencias que la precedieron, se inscribió en un movimiento internacional de reivindicación de la ciudadana que, en una coyuntura local de modernización excluyente impulsada por el orden stronista, pudo tejer una alianza con actores locales e internacionales que pregonaban un orden anticomunista.

Palabras Clave: Guerra Fría, Derecho al Sufragio Femenino, Liga Paraguaya Pro Derechos de la Mujer, Anticomunismo, Paraguay.

\begin{abstract}
:
The article seeks to the problematic interaction of political modernization and gender relations in the context of the Cold War, to explain the sociohistorical conditions that enabled the recognition of the political women's rights in Paraguay. It is proposed, specifically, to study the forms assumed by the conquest of the citizenship of a group of women from Asunción, organized in the Paraguayan League for the Rights of Women (1951-1962). It assumes that this organization, unlike other experiences that preceded it, was part of an international movement for the demand of the citizen that, in a local context of exclusionary modernization promoted by the stronist order, could weave an alliance with local and international actors that proclaimed an anticommunist order.
\end{abstract}

KeYworDs: Cold War, Right to Women's Suffrage, Liga Paraguaya Pro Derechos de la Mujer, Anticommunism, Paraguay.

\section{INTRODUCCIÓN}

A la hora de estudiar el derecho al voto de las mujeres en Paraguay bajo la dictadura de Alfredo Stroessner (1954-1989), las interpretaciones hegemónicas suelen caer en miradas que vuelven a reificar los mecanismos de la cooptación del régimen. Sin embargo, son escasos los trabajos que invierten la perspectiva y buscan identificar los actores locales e internacionales que, en el marco de la disputa ideológica brindada por la Guerra Fría, agenciaron a favor de la ampliación de los derechos políticos de las mujeres en dicho país. En efecto, Gorza y Valobra (2018) han llamado la atención sobre la vacancia de estudios que aborden una reflexión específica sobre la modernización política y las relaciones de género; de esa intersección se quiere hacer eco este trabajo.

Para ello, analizaremos cómo, en un contexto histórico específico de modernización autoritaria impulsada por el stronismo en plena Guerra Fría, la Liga Paraguaya Pro Derechos de la Mujer (LPDM) logró el reconocimiento de los derechos políticos de las mujeres (Ley No 704/1961). Partimos de la hipótesis de que 
la LPDM, a diferencia de otras experiencias que la precedieron, se inscribió en un movimiento internacional de reivindicación de la ciudadana civil y política, y pudo tejer una alianza entre actores locales y extranjeros. En el plano local, asumimos que la consagración de los derechos políticos fue posible porque la incorporación formal de las mujeres a la vida política no amenazaba la dominación de una dictadura que se legitimaba también bajo un formato democrático. Muy por el contrario, las mujeres del Partido Colorado -el partido de la dictadura stronista - tenían una larga trayectoria en el plano de la conquista de espacios partidarios: las mujeres afiliadas al coloradismo ejercieron su derecho al voto para la elección de las autoridades partidarias veinticinco años antes que fuera ley el derecho al sufragio.

Este estudio se inscribe en el campo de la sociología histórica de tiempo presente (Skocpol, 1984; Tilly, 1991), que permite analizar los hechos únicos (historia) dentro de modelos sociológicos que van más allá de la singularidad (Bonnell,1980). Si seguimos a Braudel (1968), se ubica el fenómeno en un tiempo mixto de corta, mediana y larga duración y se analizan las continuidades y discontinuidades que proveen una explicación de los factores estructurales y coyunturales que operan sobre éste. Nos centramos en dos coyunturas de cambio social — concepto privilegiado de la sociología histórica一, mirando la singularidad que, en una coyuntura específica nacional, adoptan las grandes transformaciones del capitalismo en el proceso de modernización dependiente de América Latina.

En este marco, el artículo - de carácter exploratorio aún- se inscribe en un proyecto más ambicioso que tiene como objetivo realizar una reconstrucción de la LPDM, en sus dimensiones contextuales y procesuales, reponiendo dos niveles de análisis. En el plano internacional, queremos indagar en la densidad del rol que tuvieron las organizaciones geopolíticas de la Guerra Fría y su impacto en Paraguay, específicamente, en la vinculación de la LPDM con la Comisión Interamericana de Mujeres (CIM) de la Organización de los Estados Americanos (OEA) ${ }^{2}$. En el plano político local, queremos explicar el rol que, a través de los canales institucionales, jugó el stronismo para el efectivo establecimiento del derecho al voto de las mujeres, un derecho que, a condición de no reivindicar el comunismo, ampliaba las fronteras de la legitimidad "democrática" del régimen.

La investigación se sustenta en los documentos oficiales de la LPDM, en el vocero El Feminista y en la prensa de la época.

\section{ANTECEDENTES Y ORGANIZACIONES DE MUJERES}

El estudio de las mujeres, en Paraguay, al igual que la perspectiva dominante del campo historiográfico, está centrado en el registro bibliográfico de las figuras destacadas de la vida cultural, literaria o incluso política (Barreto Valinotti, 2011; Díaz de Espada, 2005; Paredes, 2011). Asimismo, son profusos los trabajos que han reducido a las mujeres a una historia nacionalista, vinculada a su rol durante la Guerra de la Triple Alianza. Hay también largos estudios sobre las formas en que las mujeres de dicho período histórico fueron restituidas a la memoria colectiva durante la dictadura stronista (Potthast, 2010) o sobre cómo se las caracteriza e identifica en los imaginarios nacionales (Céspedes, 2016; Makaran, 2013). Principalmente, en los últimos años, y como parte de los procesos de recuperación de la memoria acerca del pasado reciente, se han hecho grandes esfuerzos por rescatar a las mujeres que habían tenido una participación activa en la vida política durante el stronismo. Su lugar, no obstante, ha sido mucho más el de víctima que el de portadora de proyectos colectivos, y el registro descriptivo no ha dejado de aparecer en clave biográfica sobre figuras notorias como Carmen Soler o Lara Castro.

Sin embargo, y gracias a trabajos pioneros (Bareiro, Soto y Monte de López Moreira, 1993; Bareiro y Soto, 1997), sabemos que las mujeres de Paraguay se destacaron en sus prácticas políticas, tanto en organizaciones internacionales como nacionales; Serafina Dávalos, por ejemplo, fue delegada oficial en el Primer Congreso Internacional de Feminismo (Buenos Aires, 1910), integró sucomisión de derecho y dictó la conferencia de clausura. Asimismo, conocemos de la existencia de colectivos de mujeres trabajadoras durante la Guerra y la 
pos Guerra de la Triple Alianza que son centrales para explicar las características que asumió el hecho bélico y la rápida reconstrucción de la economía y la sociedad paraguaya (Dalla Corte Caballero, 2018; Potthast, 2010), y que estos primeros colectivos lograron la primera ley laboral que registra Paraguay —la ley de servicio doméstico (1884) - y acompañaron de manera muy novedosa el ejercicio del quehacer público y político.

Así, aun cuando no contaban con el derecho al voto ni a postularse a cargos electivos (Monte de López Moreira, 2011a), las mujeres paraguayas tuvieron participación política y mantuvieron una constante vinculación internacional con líderes y organizaciones que compartían sus luchas. En 1920, bajo la influencia de la feminista uruguaya Paulina Luisi (está documentada su amistad con el diputado Telémaco Silvera, pionero en la defensa de los derechos de las mujeres), se formó el Centro Feminista Paraguayo (1920), que adheriría al Congreso de la Alianza Mundial para el Sufragio Femenino. Dos años después de su formación, María Felicidad González participaríade la Conferencia Panamericana de la Mujer-organizada en Baltimore por Liga Nacional de Mujeres Votantes-, durante la cual se conformaría la Liga Panamericana para la Elevación de la Mujer, devenida luego en la CIM. El texto de la conferencia fue reproducido en la revista Feminismo Internacional y elogiado por ser considerado una pieza que aportaba a la mujer paraguaya "consideraciones probatorias del grado de adelanto de su formación profesional, cultural y su espíritu de solidaridad" (Monte de López Moreira, 2011b, p. 72). González, al igual que otras mujeres que tendrían una vida política activa en ese período, formaron parte de lo que se conoce como el primer movimiento colectivo de mujeres: las concepcioneras. ${ }^{3}$ Ella fue delegada en la conferencia que se celebró en Montevideo a fines de 1933, en la que Paraguay suscribió el Tratado de Igualdad de Derechos Civiles y Políticos (Jaiven, 2009, p. 28).

Producto de esa efervescencia fueron la presentación en el parlamento del primer proyecto de divorcio, redactado por el liberal Lisandro Díaz León y apoyado públicamente por Telémaco Silvera, y la creación de la Asociación Feminista (1929), que comenzaría a debatir el Código Civil Paraguayo. Ese mismo año, Antonio Sosa, senador perteneciente al Partido Colorado, procuraría nuevamente la ampliación de los derechos civiles de las mujeres — un punto clave de la lucha de la Unión Femenina Paraguaya (Soler, 2017) —, y poco tiempo después, Telémaco Silvera — quien ya en 1919 había redactado los primeros proyectos acerca de la igualdad civil y política - incorporaría en la Convención Especial Republicana la propuesta del derecho al voto para las mujeres, que sería aprobado en noviembre de 1936 por la Comisión Central y la convención de la Asociación Nacional Republicana. Para 1945, se había formado la Comisión de Damas Coloradas, que años después se lanzaría a la calle al frente de una manifestación en reclamo de los derechos políticos y civiles para la mujer paraguaya.

En rigor, en los años 1930, la Guerra del Chaco abrió un proceso de politización interesante que afectó a grandes sectores asuncenos y, especialmente, a las mujeres. Como ha demostrado el original trabajo de Estela Mary Sosa (2010), las mujeres, durante la contienda, habían cumplido roles centrales no sólo relacionados a las actividades de reproducción económica o de residentas; las de origen urbano y letradas formaron parte de las brigadas escolares y de los servicios de enfermería en el servicio social y de sanidad. El Estado, mediante el Ministerio de Educación y Culto, también hizo lo propio poniendoen marcha la enseñanza de dactilografía, taquigrafía y redacción comercial, para que también ocuparan, transitoriamente, los puestos dejados por los hombres movilizados al frente.

Durante la siguiente década, por el contrario, la constitución autoritaria de 1940, el posterior gobierno de Higinio Morínigo (1940-1946), la guerra civil, entre otros factores, reprimirían las actividades políticas y forzarían a muchas mujeres al exilio. En rigor, recién la estabilidad del orden político sería provista con la llegada de la dictadura encabezada por Stroessner. La construcción del régimen, a partir de un proceso de modernización conservadora (Moore, 2002), seasentó sobre algunos pilares básicos: la reorganización del sistema político (modificaciones legales y constitucionales), la participación/cooptación de las élites políticas a través de los partidos (o transformismo, si se prefiere, en la conceptualización gramsciana), la mutación del Partido Colorado en partido-Estado (que además brindó el andamiaje institucional para los 
sucesivos triunfos electorales), la militarización de dicho partido (con el nombramiento de los generales como presidentes de su Junta de Gobierno) y la partidización de las Fuerzas Armadas, y un sistema eficaz de represión y cooptación que funcionó en todo el entramado social. Fue el Partido Colorado, en el marco de la democracia sin comunismo, ${ }^{4}$ el que le proveyó - por lo menos- una base institucional de legitimidad política y facilitó la organización de la dominación mediante las estructuras de los partidos, lo que posibilitó un ordenamiento político apoyado en las instituciones típicas de la democracia liberal a través de las elecciones presidenciales y legislativas y de la reforma constitucional de 1967 (Soler, 2012).

\section{Modernización Y DERECHO AL SUFRAgio FEMENINO}

El régimen stronista (1954-1989), de principio a fin, fue un orden político enmarcado en las disputas de la Guerra Fría y se inscribió en los principios rectores de un orden autoritario que pregonaba la "democracia sin comunismo". Para ello contó, desde su inicio y hasta mediados de los años 1970, con un apoyo económico inédito de los Estados Unidos, que fue mucho más allá del plano militar y se expresó en un verdadero programa de modernización del país. Todas las áreas del gobierno, al igual que todos los programas de transformación de la estructura social (desde la tenencia de tierras hasta los planes de infraestructura, pasando por los programas de reforma de los sistemas educativo y de salud), recibieron apoyo técnico y financiero externo.

Ya hacia el fin de la Guerra del Chaco, EE.UU.había desarrollado una política clara de intervención en Paraguay, especialmente por el peligro que implicaba la fuerte presencia alemana en tierra guaraní alas puertas de la Segunda Guerra Mundial. Entre 1941 y 1943, Washington abrió las llaves de las donaciones y Paraguay, que hasta el momento se había mantenido neutral, rompió las relaciones políticas y comerciales con los gobiernos de Alemania, Italia y Japón, para luego,en 1945 - cuando la guerra finalizaba-, declararse en estado de guerra con las potencias del Eje. Si, para 1939, el porcentaje de las inversiones norteamericanas en Paraguay no superaban el 17\%; para 1945, llegaban al 30\%.

Con la segunda posguerra, se volvieron notorias las nuevas formas de relación entabladas entre Paraguay y EE.UU. En el año 1945, se daría una reestructuración del vínculo de dependencia para toda América Latina, en tanto que el derrumbe de Europa, de quien la región había dependido desde su emancipación, habilitaría una nueva coyuntura con la ahora nueva potencia dominante. Así, en el marco de una relación significativa entre la nueva hegemonía mundial asumida por EE.UU. y los nuevos tipos de intervención experimentados en la región, es posible observar la apariciónde un conjunto de instituciones multilaterales que tenían como objetivo promover los procesos de integración regional y comercial en sociedades que estaban atravesando cambios sociales y culturales profundos. El Estado se convirtió así en la unidad de análisis del debate sobre el desarrollo y la modernización, en tanto debía implementar políticas públicas dirigidas a remover los obstáculos que impedían obtener el mentado progreso.

En efecto, en América Latina, el derecho al sufragio femenino fue conquistado después del estallido de la Segunda Guerra Mundial, cuando, para entonces, la Unión Panamericana llevaba dos décadas apoyando el voto de las mujeres. Entre 1933 y 1945, las Conferencias Internacionales de los Estados Americanos "generaron no menos de cuatro declaraciones y resoluciones intergubernamentales que abogaban por el derecho de la mujer a la libertad política; estas conferencias también produjeron el tratado mundial sobre los derechos políticos de la mujer en 1948" (Towns, 2010, p. 780).

Desde el cambio de siglo hasta que, en 1961, Paraguay reconoció el derecho de las mujeres a votar, en América Latina la lucha por el sufragio tuvo lugar en un contexto de aspiraciones imperiales o hegemónicas de EE.UU. en el hemisferio. Innumerables intervenciones económicas, militares y políticas sucedieron en la región.

La creación, en 1951, de la Comisión Mixta para el Desarrollo — con el fomento de Naciones Unidas y 
del Banco Mundial-permitió que Paraguay se convirtiera en el tercer beneficiario - luego de Brasil y Perú - del financiamiento económico norteamericano. Desde 1953, dada la estabilidad política lograda por el presidente Federico Chávez (1949-1954), se amplió la cooperación técnica y financiera estadounidense y se diligenciaron esfuerzos para obtener asistencia técnica por parte de las nuevas agencias de desarrollo de las Naciones Unidas y el Banco Mundial. Dependiente del comercio externo, Paraguay otorgó concesiones arancelarias a la importación, a través de acuerdos con los Estados Unidos, Argentina y Uruguay (Birch, 2011).

Derrotada la experiencia populista de la Argentina y de su aliado guaraní -el presidente Federico Chaves-, las relaciones entre EE.UU. y Paraguay entraron en una fase de cooperación sin precedentes, basada en la convergencia de las políticas de seguridad y la modernización económica de la Guerra Fría con el stronismo (Mora y Cooney, 2009). A cambio del apoyo económico y político, Paraguay acompañó la política exterior propuesta por el país norteamericano - demandando a la OEA que actuara militarmente contra Cuba - y cedió parte de su soberanía política firmando la cláusula Selden's resolution, que habilitaba la intervención directa de militares en territorio paraguayo en caso de amenaza comunista. Además, apoyó militarmente, y en los fueros diplomáticos, cada una de las medidas de EE.UU., llegando a enviar efectivos paraguayos a la República Dominicana para derrocar el gobierno de Juan Emilio Bosch Gaviño (1963) y a ofrecer colaboración, de la misma manera, en la guerra con Vietnam.

La cooperación se afianzó también en el plano cultural mediante las agencias de promoción de la cultura norteamericana. La Oficina de Asuntos Interamericanos (OCCIAA) desarrollóuna amplia actividad propagandística mediante la utilización de películas y cortometrajes que se distribuían en todo el país con muestras de la cultura, la vida y el ejército norteamericano (Mora y Cooney, 2009), y Las Amigas Norteamericanas del Paraguay, el Centro Cultural Paraguayo Americano y la militancia social de los Cuerpos de Paz se diseminaron por todo el territorio guaraní. En síntesis, Paraguay fue visto a largo plazo como un actor nodal para acompañar las políticas estadounidenses en los foros internacionales, especialmente en el sistema interamericano, participando en la formulación de programas políticos y económicos en el continente y permaneciendo como elemento confiable en el planeamiento de la seguridad hemisférica.

En este marco, debemos entender la extendida presencia que desde los años 1950 tiene Paraguay en los organismos internacionales y, en el caso que nos ocupa, en la Comisión Interamericana de Mujeres, que eligió Asunción como sede para la realización de la IX Asamblea de la Comisión Interamericana de Mujeres (del 7 al 23 de septiembre de 1953). La misma dedicó un capítulo al tratamiento de los derechos políticos ${ }^{5}$ y cuatrocientas mujeres marcharon desde la Plaza de la Independencia hasta la sede de la conferencia con las banderas de veintiún países de América (Duarte Sckell, 2017).

Para ese entonces, la CIM había logrado que se suscribieran las que serían dos normas pilares en la búsqueda de igualdad jurídica entre ambos sexos. Una de ellas fue la "Convención Interamericana sobre la Concesión de Derechos Políticos para la Mujer, la cual establecía que el derecho al voto y a ser elegido no debía restringirse por razones de sexo. De manera paralela, se suscribió la Convención Interamericana sobre Concesión de los Derechos Civiles para la Mujer, mediante la cual los Estados americanos conveníanen otorgar a las mujeres los mismos derechos civiles de los que gozaba el hombre" (Mejía Guerrero, 2012, p.192).

Cuando, en 1954, la Federación Democrática Internacional de Mujeres ${ }^{6}$ perdió el estatus consultivo en la Organización de las Naciones Unidas, acusada de ser "pantalla del comunismo", la Comisión Interamericana de Mujeres ocupó un lugar central en la agenda por la igualdad de derechos. Según el estudio de Gorza y Valobra (2018), la CIM, aunque financiada por fondos norteamericanos, tuvo un papel crítico sobre las políticas estatales y fue "el primer organismo intergubernamental en nuclearse en pos de los derechos femeninos dentro de la Organización de los Estados Americanos; también impulsaba un programa sobre derechos políticos (...) y presentó numerosos informes que recuperaban la particular situación de las americanas". Como ha estudiado Ana Lau Jaiven (2009), las sufragistas latinoamericanas mantuvieron vínculos amistosos con sus homólogas norteamericanas; con ellas desarrollaron un internacionalismo que ayudó a discutir sus pretensiones políticas y jurídicas de justicia social al interior de sus países de origen. 
En síntesis, en los años 1940, la consigna del sufragio femenino no era nueva, pero había adquirido un nuevo tono a partir de un contexto internacional favorable que auspiciaba su aprobación — una posición no ajena a la idea de afirmar la democracia frente al totalitarismo-, como se desprende de las consideraciones de la CIM, reunida en Chapultepec en 1945, que recomendaba hacer efectiva la aprobación de los derechos civiles y políticos de las mujeres acordada en la Conferencia de Lima en 1938. Pero como indica Verónica Giordano (2012), llegó recién en los años de la posguerra el momento democratizador: "Mientras que en Europa el sufragio femenino fue sancionado en 1944 en Francia y, en 1946, en Italia, en América Latina, leyes similares se proclamaron en Argentina, en 1947, y en Chile, en 1949. Y en otros países, considerados pioneros, las leyes tuvieron impacto real también en esa coyuntura" (Giordano, 2012, p. 62).

\section{Liga Paraguaya Pro Derechos de la mujer (1951-1962)}

Como hemos indicado, otras organizaciones que lucharon por la igualdad de derechos políticos de las mujeres, como la Unión Femenina del Paraguay (1936), el Consejo de Mujeres de la República del Paraguay (1940) y la Unión Democrática de Mujeres (1946),tuvieron vínculos internacionales, pero, sin lugar a dudas, la más importante organización para la conquista del voto femenino fue la Liga Paraguaya Pro Derechos de la Mujer, fundada en 1951, que en el marco de la Guerra Fría recibió el apoyo de la CIM y de la Liga de Mujeres Votantes de los EE.UU.

La liga se definía partidaria de la democracia, anticomunista y reconocía antecedentes sólo en organizaciones de género sin adscripción política partidaria. Esta organización de mujeres se inscribió en un movimiento internacional de reivindicación de la ciudadana civil y política, y logró la modificación del código civil en 1954 (Ley No 236/1954) y el otorgamiento de los derechos políticos en 1961 (Ley No 704/1961).

Alentada por la figura de Isabel Arrúa Vallejos, su primera presidenta fue Concepción Rojas Benítez, quien además de haber sido enfermera en la Guerra del Chaco, había desempeñado funciones en la embajada de EE.UU.y era representante de la CIM. Ésta, junto con Arrúa Vallejos, eran militantes del Partido Colorado. La LPDM contó también con el apoyo y la adhesión de la jurista Mercedes Sandoval de Hempel-llegó a presidirla-, quien era miembro de la International Federation of Women Lawyers ${ }^{8}$ y de Concepción Leyes de Chaves, que entre 1953 y 1957 fue electa como presidenta de la CIM con sede en Washington.

La LPDM contaba con socixs activxs y adherentes que pagaban una cuota social mensual. Tuvo su propio vocero, El Feminista - del que se editaron 34 números más uno extraordinario, entre 1953 y 1962—, como medio de difusión e intervención política. Sus redactoras explicaban que el nombre de la publicaciónse identificaba directamente con la problemática de la mujer: "Un nombre concreto y expresivo". La liga, además, mantuvo un programa de radio que se emitía semanalmente, espacio cedido por la Radio Nacional.

Arrúa Vallejos fue la directora del vocero y se desempeñó como embajadora en Chile durante parte del pinochetismo. ${ }^{9}$ Elsa Wiezell de Espínola, una prolífica poeta y participante activa del mundo artísticocultural asunceno, era la jefa de redacción. Según un estudio realizado por Monte de López Moreira (2013), El Feminista era un diario claramente anticomunista, tanto porque sus redactoras participaban en manifestaciones de ese tinte alentadas por el stronismo, como porque sus artículos denunciaban a supuestos infiltrados comunistas de la sociedad paraguaya. No obstante, es factible encontrar zonas más grises, especialmente porque escribían figuras destacadas de la cultura nacional e internacional que no siempre tomaban posiciones en contra del comunismo. En rigor, se puede percibir una pluralidad de ideas, algunas más radicales que otras, en tanto en la publicación podían convivir el nombre feminista con posturas conservadoras sobre el rol de la mujer en la sociedad. En el vocero se podía leer que el feminismo es una etapa evolutiva de lo progreso social, pero que el mismo no busca intentar contra las buenas costumbres. En rigor, "el verdadero feminismo lucha por los derechos sociales y políticos de la mujer, sin afectar la decadencia de la misma” (Acosta Elías, 1997, p. 163). 
La LPDM, según su estatuto, publicado el 15 de noviembre de 1953 en el primer número de su vocero, buscaba "iniciar una campaña de carácter nacional, tendiente a la obtención de la igualdad de derechos sin distinción de sexos, promover la conciencia colectiva en favor de los derechos de la madre y el niño y velar por las condiciones de trabajo de las mujeres empleadas y las obreras" (El Feminista, Asunción, 15 de noviembre de 1953, p. 1). Sus objetivos también se expresaban de la siguiente manera: "Persigue sembrar ideas de justicia, orden, paz y progreso dentro de los principios democráticos que nos rigen en tanto que apoya toda idea de sano nacionalismo que no excluya la fraternidad internacional ni la solidaridad continental" (El Feminista, Asunción, 15 de noviembre de 1953, p. 1).

Según la reconstrucción de Ana Barreto Vainotti (2011), la liga estuvo dirigida por mujeres coloradas e integrada por liberales. Sin embargo, la supervivencia de esta agrupación y las conquistas logradas con la ampliación de la ciudadanía se debieron a un delicado equilibro entre mantener una estrecha relación con el Partido Colorado y, a la vez, con organizaciones y figuras internacionales. La LPDM contó con el apoyo de referentes extranjeras que, al mismo tiempo, participaban en las más diversas luchas de agrupaciones femeninas, pero a las que no podría endilgárseles adscripción partidaria. De todos modos, en momentos en los que el orden político se presentaba como una amenaza para los derechos civiles, la liga desarrollaba estrategias para no sucumbir, entre ellas, nombrar a la esposa del presidente, Ligia Mora de Stroessner, como presidenta honoraria, cargo que también ocuparía Serafina Dávalos, primera mujer graduada en la Universidad de Asunción (su tesis doctoral, Humanismo, fue el primer manifiesto femenino que denunció el sistema político, jurídico y educativo paraguayo). También —a Dávalos— se la honró con ese título por haber sido la primera y única mujer en ocupar un lugar en la Corte Suprema de Justicia (1908-1909) y por haber creado una escuela comercial para niñas (1904-1905).

Sería reduccionista, no obstante, pensar que la ampliación de los derechos se debió sólo al peso de los organismos internacionales y a la mirada expectante sobre el régimen de Stroessner durante la Guerra Fría. Como en otros ámbitos ya estudiados, durante la "dictadura colorada" se llevaron a cabo manifestaciones políticas contestatarias y, muchas veces, abiertamente enfrentadas al orden "compacto" imperante. Aquí vale recordar el meeting público que alentó un puñado de mujeres a propósito de las elecciones municipales de Asunción de 1960, en las que se habían visto excluidas. ${ }^{10}$ Parte de la acción colectiva consistió en distribuir panfletos a los transeúntes y en los ómnibus que iban al interior del país. En las consignas se podía leer: "Los extranjeros votan ¿Por qué las mujeres no votamos?” / "Dicen que somos democráticas, pero los derechos políticos no son para las mujeres".

Parte de la protesta colectiva se vio alentada por la relación entre una periodista española de la revista $L i f e,{ }^{11}$ que visitó Asunción en esos años, y la jurista Sandoval de Hempel, que además de pertenecer a la liga ocupaba - como ya indicamos - cargos destacados en organismos internacionales. Lo cierto es que el reclamo, que le valió la detención policial por algunos días a dos de las manifestantes, tuvo repercusión internacional. "La Prensa de Buenos Aires y La Nación publicaron la carta de petición firmada por los intelectuales y políticos paraguayos (entre los que se encontraba la presidenta de la LPDM) en la que pedían al dictador el reconocimiento del voto" (Mora, 1997, p. 119).

En un clima internacional y local sensibilizado, en el contexto de la Guerra Fría y de una dictadura que quería proyectarse como una "democracia perfecta" (es decir, sin conflicto social), el 5 de julio de 1961, Stroessner promulgó la Ley No 704, aprobada el 30 de junio por el Poder Legislativo. Su primer artículo reconocía a la mujer paraguaya los mismos derechos y las mismas obligaciones políticas que al hombre, y esta noticia tuvo repercusiones internacionales y locales importantes. El vocero de la organización publicaba:

La Comisión Interamericana de Mujeres destacó desde el exterior la posición singular del Paraguay entre sus hermanos de América, la de ser el único que faltaba en la lista de los que habían reconocido la completa igualdad. También, dentro del país, las mujeres afiliadas a los partidos políticos hicieron oír su voz, mientras algunos distinguidos intelectuales se sumaban a la defensa de los derechos femeninos (El Feminista, edición extraordinaria, Asunción, 15 de agosto de 1962, p. 15). 
La CIM, en una nota que "enaltece su verdadera misión”, se dirigió al ministro de Relaciones Exteriores paraguayo, Raúl Sapena Pastor, manifestando que "la mujer paraguaya, que tan brillantes y heroicas páginas tiene escritas en la historia de su país, debe haber recibido la noticia de este reconocimiento con la satisfacción que da quién ha luchado por el triunfo de la misma" (Patria, Asunción, 26 de julio de 1961, p.5).

Los diarios locales de la época, de manera dispar, reflejaron la noticia de la sanción del derecho al voto para las mujeres. La Tribuna, el diario más tradicional, apenas le dedicó un pequeño recuadro; el diario el País, días antes de la promulgación, había reproducido el discurso a favor de los derechos políticos de las mujeres pronunciado por el sociólogo y parlamentario colorado Manuel Frutos Pane y, desde el 24 de junio, había seguido con atención el desarrollo del tratamiento del proyecto elevado por el Poder Ejecutivo. El diario Patria, órgano de prensa oficial de la dictadura stronista, también reproducía el discurso del parlamentario colorado, pero bajo el título de "Una conquista más de los derechos políticos", y aclaraba en la nota que se trataba de "una conquista más del coloradismo en el poder". Sin duda, el diario de la dictadura fue el que más cobertura ofreció a la nueva conquista; ese medio era, tanto como su subtitulo versaba, "vocero de la Junta de Gobierno del Partido Colorado” y, en tanto voz del stronismo, intentó que éste se apropiarade la ampliación de los derechos políticos paracapitalizarla a su favor. Las comisiones de mujeres de las secciones enviaron varias notas de agradecimiento que fueron reproducidas por ese medio periodístico.

En la convención partidaria colorada del 5 de julio de 1961, la recientemente sancionada ley de los derechos políticos ocupó un lugar privilegiado y recibió elogios de los principales dirigentes "legítimo orgullo del partido y del Gobierno nacional” (Patria, Asunción, 5 de julio de 1961, p. 7). Allí, se decidió encomendar a la comisión política del partido "un estudio exhaustivo" del impacto que la nueva ley tendría en la participación juvenil y femenina del coloradismo.

El partido colorado, mediante manifestaciones en el espacio público, publicitaba lo que mostraba como su triunfo: "Las damas coloradas protagonizaron ayer fervorosa y emotiva jornada de civismo", titulaba Patria el 26 de julio de 1961. Según el vocero, las secciones femeninas de la coloradas, y las correligionarias en general, se reunieron en la Casa de los Colorados para reconocer a la Junta del Gobierno y a la Comisión Central Femenina del partido - presidida por Bienvenida de Sánchez-, y para agradecer los esfuerzos desplegados por las autoridades partidarias en favor de la promulgación de la ley de los derechos políticos de la mujer paraguaya. El presidente de la Junta, Bernardino Gorostiza, y su esposa, Carmen de Gorostiza, también participaron. "La caravana", como tituló Patria, se movilizóhacia el Panteón Nacional de los Héroes, donde se venera, además, a la Virgen de Asunción. Allí, se depositaron ofrendas florales al Mariscal del Ejército (el prócer nacional por excelencia, Francisco Solano López) como testimonio de agradecimiento por el reconocimiento de los derechos políticos.

El acto prosiguió en la residencia presidencial, el Palacio de los López, con una "selecta delegación de damas coloradas, representantes de las 25 secciones coloradas". En esta oportunidad las recibió la "señora esposa”, Eligia Mora de Stroessner, y el presidente Stroessner. La presidenta de la Sección Femenina No 15 se dirigió al mandatario diciendo: "Las damas coloradas nos comprometemos a colaborar con vuestro Gobierno, desde todos los lugares que nos toca actuar: como madre, novia e hija, como empleada, obrera, profesional y política", y también indicó que la ley no es una mera fórmula intrascendente, sino que "es sabido que cuando una mujer se dispone a defender una causa tomada, lo hace con tenacidad inquebrantable" (Patria, Asunción, 26 de julio de 1961, p. 2).

Stroessner no perdió la oportunidad de dirigirse al público femenino, interpelando al colectivo como "damas correligionarias o compañeras que han merecido la consagración de los derechos políticos" (Patria, Asunción, 26 de julio de 1961, p. 2). El acto fue trasmitido en cadena nacional por las emisoras de radio y buena parte de su discurso lo dedicó a vincular la nueva condición política de las mujeres con la relectura de un pasado nacionalista, que se consagraba en la figura de Elisa Lynch, cuyos restos fueron repatriados del cementerio parisino del Père Lachaise pocos días después de que la ley fuera sancionada. 
El 29 de julio se volvió a realizar un acto político "de gratitud a la mujer colorada", que tuvo expresión multitudinaria. El presidente del partido, el ya mencionado Bernardino Gorostiza, impulsó la participación femenina en el partido:

"Las exhorto patrióticamente a que ingresen las que aún no ingresaron en las tradicionales asociaciones políticas de nuestra patria [...] Muy pronto las veremos a nuestras abnegadas y aguerridas compañeras de la lucha ingresar en la Junta de Gobierno y en las comisiones sectoriales para compartir sus luces y su sagacidad al lado de los hombres [...] y muy pronto las veremos también al lado de los hombres y al igual que ellos en el Parlamento, en las juntas municipales y en algunas funciones del Gobierno nacional" (Patria, Asunción, 29 de julio de 1961, p. 2).

El acto, realizado en un teatro municipal, contó también con la presencia del ministro de Justicia y Trabajo, Juan Chaves - otro personaje de peso en el régimen stronista—, y algunas mujeres del partido participaron de la oratoria. Hubo coros, bailes y orquesta y fue vivido como una fiesta partidaria y nacional.

Poco tiempo después de sancionada la ley del derecho al sufragio, la LPDM se preguntaba:

“QQué debemos hacer las mujeres? En síntesis, lo siguiente: capacitarnos cada vez más para la acción cívica; pensar en la solución de los problemas de la colectividad, aportando ideas sanas y desechando los fines egoístas; predicar y practicar la unión y la tolerancia; dentro de la moral y las buenas costumbres; tener como mira la defensa de la paz y la democracia; ayudar en todo lo posible al engrandecimiento de la patria" (El Feminista, edición extraordinaria, Asunción, 15 de agosto de 1962, p. 15).

La liga se disolvió en 1961 y El Feminista se siguió publicando hasta 1962. Parte de la organización se mantuvo funcionando bajo el nombre de Liga Paraguaya de los Derechos de la Mujer, pero más que un espacio para la emancipación, se convirtió en un espacio de socialización de género vinculado al régimen stronista. Podemos decir que, una vez lograda su principal bandera de reivindicación, la liga perdió su identidad principal y se transformó, en palabras de Graziella Corvalán, en "un mecanismo de control destinado a mantener la situación de las mujeres y una institución más de reclutamiento y socialización del partido en el Gobierno" (Corvalán, 1985, s/p). En rigor, hubo que esperar a la caída de Stroessner para poder escribir otro capítulo acerca de las luchas emancipadoras feministas.

\section{Preguntas de cierre}

La LPDM hizo del contexto internacional favorable a la modernización y ampliación de derechos y de un régimen político preocupado por hacer de la democracia sin comunismo su bandera de legitimidad política, la condición de posibilidad para la ampliación del estatus civil y político de las mujeres. Como sabemos, las dictaduras en América Latina no fueron ajenas a ejercer la dominación bajo "formato representativo", y las elecciones periódicas y la vigencia del Congreso fueron elementos promovidos por los gobiernos autoritarios para legitimar su accionar represivo y excluyente.

Una discusión que ha mantenido ocupada a buena parte de la literatura sobre el tema es, como primera pregunta, el hecho de cómo dimensionar el peso de la lucha de las mujeres por los derechos y la apropiación que la dictadura hizo de dicha conquista. La segundapregunta inmediata que surge de la sanción de la ley es si ésta posibilitó una transformación de la vida política de las mujeres y si tuvo un reflejo relativamente inmediato en la configuración de los partidos políticos. Claro está que la igualdad jurídica de las mujeres formaba parte de la agenda antes de la llegada del stronismo. La especificidad es que durante dicha dictadura se coaligaron factores externos e internos que hicieron posible la sanción del derecho femenino al voto. Para el proyecto stronista no era algo conflictivo ni disruptivo la incorporación de las mujeres a la vida política, como tampoco lo fue, en 1870, el derecho al sufragio para todos los hombres mayores de 18 años, sin distinción de raza, propiedad ni alfabetismo; en ambos contextos históricos se trataba de actores excluidos de la vida política, no "peligrosos" para el orden social. Sin embargo, una vez conquistados los derechos, la participación se fue ampliando y modificó la vida pública. 
El 10 de febrero de 1963, las mujeres inscriptas en los padrones pudieron por primera vez ejercer el derecho al sufragio. Estaban habilitadxs unos 714.000 votantes y los escrutinios dieron ganador a Stroessner con 438.043 votos contra los 40.313 obtenidos por el segundo, Ernesto Gavilán. Una distribución porcentual similar, con diferentes opositores eleccionarios, se repetiría en los siguientes comicios de los años 1968, 1973, 1978, 1983 y 1988 y otorgaría reiteradamente la victoria al dictador Stroessner y a sus colaboradores, quienes ocuparían, en mayoría, los escaños del Congreso.

En 1963, también por primera vez en la historia del país, y dada la temprana participación femenina que se diera en el Partido Colorado,dos mujeres — de dicha organización-, Bienvenida de Sánchez y Dolores de Miño, llegarían al parlamento.Durante la dictadura de Stroessner, esa tendencia se mantuvo, pero llegó a ampliarse a cuatro mujeres en algunos períodos cuando se pasó de un sistema cameral a otro bicameral (1973-1978 y 1988-1989). ${ }^{12}$ En 1989, tras la caída de Stroessner, asumióla primera mujer como ministra y la cantidad de parlamentariasse fue ampliando hasta constituir el 6\% de la representación total de las cámaras.

En 1991, los partidos comenzaron un proceso de democratización de género e incorporaron las cuotas en sus estatutos; coherente con su historia de apertura a la participación política de la mujer, el Partido Colorado fue entonces también el primero en hacerlo.Luego, con la sanción de una nueva ley electoral en democracia (1996), se estipularon cuotas mínimas de un 25\% para las listas que se presenten en las elecciones internas de los partidos políticos.

Durante la apertura democrática, la representación fue aumentando hasta lograr, en el período 2013-2018, nueve bancadas para diputadas y doce para senadoras (el 16\% y el 12\% del total de la representación respectivamente en cada cámara). Dicha representación por género se encuentra muy por debajo del promedio regional, que actualmente asciende al $23,27 \%$ en las cámaras únicas o de diputados y al 22,5\% en las cámaras de senadores.

Las mujeres también llegaron a competir por la presidencia y vicepresidencia de la nación. En 1993, María Victoria Brusquetti fue candidata a vicepresidenta por el Partido Encuentro Nacional; en 2008, Blanca Ovelar fue candidata a presidenta por el Partido Colorado, y en 2013, Lilian Soto fue candidata, también a presidenta, por la formación partidaria feminista Kuña Pyrenda. Sin embargo, esta participación creciente no les valió aún para ocupar lugares en las estructuras de poder en proporciones significativas: el porcentaje de mujeres en el Poder Ejecutivo de las gobernaciones y los municipios del Paraguayno supera el 6\%.

Finalmente, la modernización dependiente muestra en América Latina procesos siempre inacabados: al tiempo que habilita momentos emancipatorios, los repone siempre de manera excluyente. El tipo de relación dependiente entre política local e instituciones internacionales se expresa así hasta el presente.

\section{REFERENCIAS}

Acosta Elías, M. (1997). Planteamientos y debates en la prensa feminista de la primera mitad del siglo XX en el Paraguay (pp. 157-167). En L. Bareiro y C. Soto, Ciudadanas. Una memoria inconstante. Caracas: Nueva Sociedad.

Bareiro, L. y Soto, C. (1997). Ciudadanas. Una memoria inconstante. Caracas: Nueva Sociedad.

Bareiro, L.; Soto, C. y Monte de López Moreira, M. (1993). Alquimistas, documentos para otra historia de las mujeres. Asunción: Centro de Documentación y Estudio.

Barreto Valinotti, A. (2011). Mujeres que hicieron historia en el Paraguay. Asunción: Servilibro-Ateneo Cultural.

Birch, M. (2011). Estabilidad política y rezago económico. El gobierno de Federico Chávez 1949-1954 (pp.148-179). En F. Masi y D. Borda (editores), Estado y economía en Paraguay 1870-2010. Asunción: CADE.

Bonnell, V. (1980). The uses of theory, concepts and comparison in historical sociology. Comparative Studies in Society and History, 2(2), 156-173.

Braudel, F. (1968). La historia y las ciencias sociales. Madrid: Alianza.

Céspedes, R. (2016). Imaginarios, memoria y tiempo en Paraguay. Asunción: FLACSO-Paraguay. 
Corvalán, G. (1985). La acción colectiva de la mujer urbana en el Paraguay. Asunción: Centro Paraguayo de Estudios Sociológicos.

Dalla Corte Caballero, G. (2018). Mujeres, sociedad y economía de la república del Paraguay. Anuario digital, 194-216. Recuperado de http://anuariodehistoria.unr.edu.ar/ojs/index.php/Anuario/index

de Haan, F. (2017). La Federación Democrática Internacional del Mujeres (FDIM) y América Latina, de 1945 a los años setenta. (pp. 17-45). En A. M. Valobra y M. Yusta Rodrigo, Queridas camaradas. Historias iberoamericanas de mujeres comunistas, 1935-1975. Buenos Aires: Editorial Miño y Dávila.

Díaz de Espada, S. (2005). 500 mujeres contemporáneas. Asunción: Servilibro.

Duarte Sckell, J. A. (2017).The attainment of women's civil and political rights in Paraguay. Oxford: University of Oxford Latin American Centre.

Giordano, V. (2012). Instantáneas del camino de los derechos políticos femeninos en la construcción del orden en América Latina. Revista ciencias sociales, 82, 61-65.

Gorza, A. y Valobra, A. M. (2018).¿Mujeres modernas para la modernización politica? Debates sobre la participación de las mujeres en la política, 1955-1966.

Jaiven, A. L. (2009). En la búsqueda por la igualdad de derechos para las mujeres. Temas de mujeres, 5(5), 17- 32.

Makaran, G. (2013). La imagen de la mujer en el discurso nacionalista paraguayo. Latinoamérica, 52,43-75.

Martínez, O. y Monte de López Moreira, M. (1999). Dios Proteja Destino Patria. Las Concepcioneras de 1901. Asunción: Centro de Documentación y Estudios.

Mejía Guerrero, L. P. (2012). La Comisión Interamericana de Mujeres y la Convención de Belém do Pará. Impacto en el Sistema Interamericano de Derechos Humanos. Revista del Instituto Interamericano de Derechos Humanos, $56,189-213$.

Monte de López Moreira, M. (2011a). Antecedentes de los derechos políticos de las mujeres en Paraguay (pp. 29-45). En M. Monte de López Moreira y C. Soto, Al fin ciudadanas. Asunción: Centro de Documentación y Estudios.

Monte de López Moreira, M. (2011b). Cronología Antecedentes de los derechos políticos de las mujeres en Paraguay (pp. 45-103).En M. Monte de López Moreira y C. Soto, Al fin ciudadanas. Asunción: Centro de Documentación y Estudios.

Monte de López Moreira, M. (2013). Los ecos de la prensa femenina en la primera mitad del siglo XXI (pp. 205-218). En J. M. Casal y T. Whigham (ed.), Paraguay, investigaciones de historia social y política. Asunción: Tiempo de Historia.

Moore, B. (2002). Los origenes sociales de la dictadura y de la democracia.El señor y el campesino en el mundo moderno. Barcelona: Ediciones Península.

Mora, M. I. (1997). La liga de las mujeres (pp. 118-121). En L. Bareiro y C. Soto, Ciudadanas. Una memoria inconstante. Caracas: Nueva Sociedad.

Mora, F. y Cooney, J. (2009). El Paraguay y los Estados Unidos. Asunción: Intercontinental.

Paredes, R. (2011). Mujeres rebeldes por la patria. Asunción: SM-Servilibro.

Potthast, B. (2010). La mujer en la historia del Paraguay (pp. 317-336). En I. Telesca (coord.), Historia del Paraguay. Asunción: Taurus.

Skocpol, T. (1984). Los Estados y las revoluciones sociales. México: Fondo de Cultura Económica.

Soler, L. (2012). La larga invención del golpe. El stronismo y el orden político paraguayo. Buenos Aires: Imago Mundi.

Soler, L. (2017). El Partido Comunista y la Unión Femenina del Paraguay en la coyuntura de los años 1930 (pp. 113-132). En A. M. Valobra y M. Yusta Rodrigo, Queridas camaradas. Historias iberoamericanas de mujeres comunistas, 1935-1975. Buenos Aires: Editorial Miño y Dávila.

Soler, L. y González Bozzolasco, I. (2017). Normalizar el Paraguay. La cruzada mundial de la amistad y su inserción internacional (1958-2011). Nuevo mundo, mundos nuevos, Recuperado de http://nuevomundo.revues.org/705 45

Sosa, E. M. (2010). El papel de las mujeres paraguayas en la Guerra del Chaco (1932-1935): relaciones de género en contexto bélico. Posadas: Universidad Nacional de Misiones. 
Tilly, C. (1991). Grandes estructuras, procesos amplios, comparaciones enormes. Madrid: Alianza.

Towns, A. (2010). The Inter-American Commission of Women and Women's Suffrage, 1920-1945. Journalof Latin American studies, 42, 779-807.

\section{Documentos ReLevados}

Actas de la IX Asamblea de la Comisión Interamericana de Mujeres (del 7 al 23 de septiembre de 1953, Asunción) Estatuto de la Liga Paraguaya Pro Derechos de la Mujer, en el vocero El Feminista, No 1, 15 de noviembre de 1953. Ley de los Derechos Civiles de la Mujer (Ley N²36/54, del 26 de agosto de 1954) Ley de los Derechos Políticos de las Mujeres (Ley $N^{\circ} 704 / 61$, del 5 de julio de 1961) Vocero El Feminista

Diario Patria

Diario La Tribuna

\section{Notas}

1 Una primera versión de este artículo fue presentada como ponencia y recibió una mención en la Universidad Nacional de la Plata en las Vo Jornadas CINIG de Estudios de Género y Feminismos y III ${ }^{\circ}$ Congreso Internacional de Identidades en 2018.

2 Los primeros antecedentes datan de 1923, en el marco de la V Conferencia Internacional Americana de la Unión Panamericana de Naciones. Finalmente, la CIM se creó el 18 de febrero de 1928 en La Habana, en el marco de la Conferencia Internacional Americana, y fue el primer organismo a nivel mundial destinado a velar por los derechos de las mujeres. Se integraría con delegadas de veintiún países. Las siete fundadoras fueron la estadounidense Doris Stevens - quien fuera designada presidenta—, la argentina Ernestina A. López de Nelson, la colombiana María Elena de Hinestrosa, la haitiana Télegny Mathon, la panameña Clara González, la salvadoreña María Álvarez de Guillén Rivas y la venezolana Lucila Luciani de Pérez Díaz.

3 En 1901, se registró una protesta de treinta y seis mujeres de Villa Concepción —la zona económicamente más rica y pujante de la época-, quienes se organizaron en torno a una denuncia de fraude en la elección del senador José Segundo Decoud. Mediante un telegrama al Senado Nacional, expresaron su disconformidad acerca de cómo dicha institución parlamentaria había resuelto el caso. Un trabajo minucioso de reconstrucción histórica puede leerse en Martínez y Monte de López Moreira (1999).

4 La lucha anticomunista adoptó estatus jurídico al sancionarse la Ley de Defensa de la Democracia (Ley No 294, del 17 de octubre de 1955), que regulaba las penas que se aplicarían a quienes "difundieran la doctrina comunista o cualesquiera otra doctrina o sistema que se propusiera destruir o cambiar por la violencia la organización democrática republicana de la Nación".

5 Ver la página 45 del Acta de la Asamblea de la comisión.El tratamiento de este punto merecería otro trabajo, por la extensión y la profundidad del análisis que debería realizarse.

6 En 1945, impulsada por el comunismo y vastos sectores, surge en París la Federación Democrática Internacional de Mujeres, cuyo fin era convertirse en vocera de la paz, erradicar el fascismo y mejorar las condiciones de vida las mujeres, tomando como modelo a la URSS y a las nuevas democracias. Ver Hann (2017).

7 El mismo año que se creó la LPDM, la Cámara de Representantes ratificó la "Convención Interamericana sobre la Concesión de los Derechos Civiles a la Mujer”, suscrita por Paraguay en la IX Conferencia Internacional Americana celebrada en Bogotá, Colombia, en 1948. Cuando dicha ratificación acababa de ser aprobada, el parlamentario colorado, Dr. Hipólito Sánchez Quell, presentó el que sería el tercer proyecto de igualdad legal para mujeres y hombres, fundamentado en el Art. 9 del programa del Partido Colorado, que proponía la "igualdad civil y política de ambos sexos".

8 Es una organización internacional no gubernamental (ONG) creada en 1944 en la Ciudad de México que obtuvo el estatus Consultivo de la ONU en 1954

9 En una foto de la revista Yuacjhu. Revista internacional de la Cruzada Mundial de la Amistad, perteneciente a una organización civil anticomunsita (Soler y González Bozzolasco, 2017) se la puede ver junto al presidente de Chile, Agusto Pinochet. La foto acompaña una nota sobre el cincuentenario de la Fundación de la CIM 
10 En un estudio sobre la UniónFemenina del Paraguay (Soler, 2017), hemos reparado en que la lucha por la conquista de los derechos políticos se restringía al ámbito municipal. No es casual entonces que la manifestación se realizara en el contexto de las elecciones para elegir autoridades locales.

11 Las fuentes consultadas no indican el nombre de esta periodista.

12 A pesar de que la Constitución de 1967 consagraba la representación proporcional en su artículo 111, se mantuvo en vigencia un criterio por el cual el partido que obtuviese más votos en las elecciones obtenía automáticamente dos tercios de los escaños parlamentarios. Tal mecanismo garantizaba el dominio del Partido Colorado, a la vez que promovía la división de las fuerzas de la oposición. 